\title{
On the electrostatic potential in the scrape-off layer of magnetic confinement devices
}

\author{
J Loizu, P Ricci, F D Halpern, S Jolliet and A Mosetto \\ Ecole Polytechnique Fédérale de Lausanne (EPFL), Centre de Recherches en Physique des Plasmas \\ Association Euratom-Confédération Suisse, CH-1015 Lausanne, Switzerland \\ E-mail: joaquim.loizu@epfl.ch
}

Received 5 July 2013, in final form 28 August 2013

Published 28 November 2013

Online at stacks.iop.org/PPCF/55/124019

\begin{abstract}
The mechanism regulating the equilibrium electrostatic potential in the scrape-off layer (SOL) of magnetic confinement devices is elucidated. Based on a generalized Ohm's law and the boundary conditions at the magnetic presheath entrance, an analytical expression for the equilibrium electrostatic potential is derived. Results imply that the relative importance of the plasma dynamics at the sheath and far away from the wall in setting the value of the electrostatic potential depends on the density and temperature drops that are established between the two regions. Global, three-dimensional fluid simulations of tokamak SOL turbulence in a simple configuration are performed, confirming the validity of our predictions. The results presented here are general and can be applied to other open-field-line configurations, including linear devices and simple magnetized toroidal devices.
\end{abstract}

(Some figures may appear in colour only in the online journal)

\section{Introduction}

Understanding the plasma dynamics in the scrape-off layer (SOL) of magnetic confinement devices, namely the region where the magnetic field lines are open and the plasma interacts with the solid walls, is crucial for the achievement of magnetic fusion. In fact, the SOL determines the boundary conditions for the core plasma, and controls the plasma refuelling, heat losses and impurity dynamics, largely governing the fusion power output of the entire device [1,2]. In particular, the origin of a high-confinement regime, where turbulence is suppressed and the formation of a plasma pedestal is observed, is still not fully understood and is the subject of intense theoretical and experimental research. There is nonetheless increasing experimental evidence for the role of the SOL in regulating the low-to-high ( $\mathrm{L}-\mathrm{H})$ confinement mode power threshold as well as the toroidal rotation profiles of the entire plasma volume [3].

An essential quantity for the understanding of mean flows and pedestal formation during the $\mathrm{L}-\mathrm{H}$ transition in a magnetically confined plasma is the self-generated radial electric field. Typically, the relation $e \phi \sim 3 T_{\mathrm{e}}$ is invoked for the SOL, where $\phi$ is the electrostatic potential and $T_{\mathrm{e}}$ is the electron temperature, thus leading to an estimate of the radial electric field as $E_{r} \sim-3 \partial_{r} T_{\mathrm{e}} / e$ [4]. This relation is based on the assumption that $\phi$ in the SOL is governed by its value at the sheath, the region where the plasma interacts with the wall. More precisely, if one uses Bohm's law for the sheath parallel current [4], $j_{\|}=e n c_{\mathrm{s}}\left[1-\exp \left(\Lambda-e \phi / T_{\mathrm{e}}\right)\right]$, then ambipolar outflow in the parallel direction imposes $e \phi=\Lambda T_{\mathrm{e}}$ at the sheath edge. Here $n$ is the plasma density at the sheath edge, $c_{\mathrm{s}}$ is the plasma sound speed and $\Lambda=\log \left[\sqrt{m_{\mathrm{i}} /\left(2 \pi m_{\mathrm{e}}\right)}\right] \approx 3$ for hydrogen plasmas. However, the generality and correctness of this result remain unclear. For instance, as we show later the parallel dynamics far from the walls can also determine the value of $\phi$, e.g. through the electron adiabaticity condition. As a matter of fact, the mechanism setting the value of the plasma potential in the SOL of magnetic confinement devices remains an open and very general issue, as it arises in all openfield-line magnetized plasma configurations, including linear devices and simple magnetized toroidal devices.

In the present article we address this question by means of an analytical model that describes the electrostatic potential in an open-field-line configuration. We provide a general analytical relation between the equilibrium electrostatic 
potential and the equilibrium electron temperature and plasma density, $\bar{\phi}=\bar{\phi}\left(\bar{T}_{\mathrm{e}}, \bar{n}\right)$, which includes the combined effect of both the sheath and the main SOL plasma dynamics (the overbar denotes time-averaged values). The analytical results suggest that, depending on the density and temperature drops established between the two regions, one mechanism can dominate over the other. This implies that the radial electric field in the SOL may be determined by different mechanisms depending on the particular regime of operation, i.e. the sheath-limited regime as opposed to the detached regime. In order to confirm our analytical predictions, we perform numerical simulations of SOL turbulence using GBS [5], a global, three-dimensional, flux-driven, full- $n$, two-fluid code based on the drift-reduced Braginskii equations and a proper set of boundary conditions to describe the sheath dynamics.

This paper is structured as follows. After the introduction, in section 2 we derive an analytical expression relating $\bar{\phi}$ with $\bar{T}_{\mathrm{e}}$ and $\bar{n}$ in the SOL. The GBS code is described in section 3 with the simulation results and the confirmation of the theoretical predictions. The conclusions and an outlook follow.

\section{Analytical model for the value of $\bar{\phi}$ in an open-field-line configuration}

An electrostatic drift-reduced fluid model is suitable to describe the SOL [6,7], and we use it here for the description of the plasma dynamics. In fact, the electron mean free path is much smaller than the connection length, $\lambda_{\mathrm{e}} \ll L_{\|}$, and trapped particles play a minor role since $v_{\mathrm{i}, \mathrm{e}}^{*} \gg 1$ [8]. Of crucial importance for the SOL is the description of the plasma losses at the vessel, which are determined by sheath physics [4]. While fluid models that are based on the drift approximation cannot describe the plasma-wall transition region, boundary conditions have been derived to supply the sheath physics to such models [9]. We remark that a fluid approach is in general reasonable for open-field-line configurations, since the plasma is not well confined and thus remains always sufficiently cold for kinetic effects to play an important role.

Within a drift-reduced fluid model, the momentum equation for the electrons in the parallel direction leads to a generalized Ohm's law,

$$
m_{\mathrm{e}} n \frac{\mathrm{d} V_{\| \mathrm{e}}}{\mathrm{d} t}=e n \nabla_{\|} \phi-\nabla_{\|} p_{\mathrm{e}}-0.71 n \nabla_{\|} T_{\mathrm{e}}+e n v j_{\|},
$$

where $V_{\| \mathrm{e}}$ is the electron parallel velocity, $\mathrm{d} / \mathrm{d} t=\partial_{\mathrm{t}}+V_{\| \mathrm{e}} \nabla_{\|}+$ $\boldsymbol{v}_{E} \cdot \nabla_{\perp}$ is the Lagrangian derivative, $\boldsymbol{v}_{E}$ is the $\boldsymbol{E} \times \boldsymbol{B}$ velocity, $p_{\mathrm{e}}=n T_{\mathrm{e}}$ is the electron scalar pressure, $j_{\|}=e n\left(V_{\| \mathrm{i}}-V_{\| \mathrm{e}}\right)$ is the parallel current and $v$ is the plasma resistivity. The absence of the electron diamagnetic drift in the material derivative is due to the so-called diamagnetic cancellation, which arises from the lowest-order term in the pressure tensor [10]. Higher-order terms in the pressure tensor, which correspond to the effect of finite electron viscosity, are smaller than the other terms by a factor $\lambda_{\mathrm{e}} / L_{\|} \ll 1$ and thus are neglected. While the electron inertia and the resistivity terms in equation (1) can play an important role in the plasma dynamics (e.g., they can make drift waves unstable by breaking the electron adiabaticity), the equilibrium profiles do not depend significantly upon those. In fact, the ratio of the inertia term to the parallel electric field term is of the order of the mass ratio $m_{\mathrm{e}} / m_{\mathrm{i}} \ll 1$. Similarly, the resistive term is negligible as long as the electron mean free path is not too small, namely if $\sqrt{m_{\mathrm{e}} / m_{\mathrm{i}}} \ll \lambda_{\mathrm{e}} / L_{\|} \ll 1$. Timeaveraging equation (1) and neglecting inertia and resistivity, we are led to an equation balancing the parallel electric field force with the parallel pressure and thermal forces,

$$
e \nabla_{\|} \bar{\phi}-\frac{\bar{T}_{\mathrm{e}}}{\bar{n}} \nabla_{\|} \bar{n}-1.71 \nabla_{\|} \bar{T}_{\mathrm{e}} \simeq 0,
$$

where we have assumed that $\overline{\left(T_{\mathrm{e}} / n\right) \nabla_{\|} n} \simeq\left(\bar{T}_{\mathrm{e}} / \bar{n}\right) \nabla_{\|} \bar{n}$. While equation (2) is valid along each magnetic field line in the SOL, it breaks down at the magnetic presheath entrance, where the drift-reduced approximation is violated [9]. Integrating equation (2) along the parallel direction $z$, from $z=-L_{\|} / 2$ to $z$ (where $L_{\|}$is the parallel connection length, and $z=0$ is defined half way between the two ends of a field line), we can write

$e \bar{\phi}(z)=e \phi^{-}+1.71\left[\bar{T}_{\mathrm{e}}(z)-T_{\mathrm{e}}^{-}\right]+\int_{-L_{\|} / 2}^{z} \frac{\bar{T}_{\mathrm{e}}}{\bar{n}} \frac{\partial \bar{n}}{\partial z^{\prime}} \mathrm{d} z^{\prime}$,

where we denote $\phi^{ \pm}=\bar{\phi}\left( \pm L_{\|} / 2\right)$ the electrostatic potential at the magnetic presheath entrance at both ends of a field line. Analogously, $\bar{\phi}(z)$ can be obtained by integrating equation (2) from $z=+L_{\|} / 2$ to $z$, i.e.

$e \bar{\phi}(z)=e \phi^{+}+1.71\left(\bar{T}_{\mathrm{e}}(z)-T_{\mathrm{e}}^{+}\right)+\int_{+L_{\|} / 2}^{z} \frac{\bar{T}_{\mathrm{e}}}{\bar{n}} \frac{\partial \bar{n}}{\partial z^{\prime}} \mathrm{d} z^{\prime}$.

We can estimate $\bar{\phi}(z)$ as the average of the values given by equations (3) and (4),

$$
\begin{aligned}
e \bar{\phi}(z)= & \frac{1}{2}\left(e \phi^{+}+e \phi^{-}\right)+1.71\left[\bar{T}_{\mathrm{e}}(z)-\frac{1}{2}\left(T_{\mathrm{e}}^{+}+T_{\mathrm{e}}^{-}\right)\right] \\
& +\frac{1}{2}\left[I^{+}(z)+I^{-}(z)\right],
\end{aligned}
$$

where we have defined

$$
I^{ \pm}(z)=\int_{ \pm L_{\|} / 2}^{z} \frac{\bar{T}_{\mathrm{e}}}{\bar{n}} \frac{\partial \bar{n}}{\partial z^{\prime}} \mathrm{d} z^{\prime} .
$$

In order to progress analytically, we write

$$
I^{ \pm}(z)=\sigma_{0}^{ \pm} \int_{ \pm L_{\|} / 2}^{z} \frac{\partial \bar{n}}{\partial z^{\prime}} \mathrm{d} z^{\prime}
$$

where

$$
\sigma_{0}^{ \pm}=\frac{\int_{ \pm L_{\| / 2}}^{z} \sigma\left(z^{\prime}\right) \frac{\partial \bar{n}}{\partial z^{\prime}} \mathrm{d} z^{\prime}}{\int_{ \pm L_{\|} / 2}^{z} \frac{\partial \bar{n}}{\partial z^{\prime}} \mathrm{d} z^{\prime}}
$$

and we have introduced the function $\sigma(z)=\bar{T}_{\mathrm{e}}(z) / \bar{n}(z)$. In the case that $\sigma(z)$ does not vary significantly along the field line, we can assume $\sigma_{0}^{+}=\sigma_{0}^{-}=\sigma_{0}$ and the value $\sigma_{0}$ can be estimated, for example, as $\sigma_{0} \simeq\langle\sigma\rangle_{z}$, where $\langle\cdot\rangle_{z}$ denotes the average along the field line. Equation (5) thus becomes

$$
\begin{aligned}
e \bar{\phi}(z)= & \frac{1}{2}\left(e \phi^{+}+e \phi^{-}\right)+1.71\left[\bar{T}_{\mathrm{e}}(z)-\frac{1}{2}\left(T_{\mathrm{e}}^{+}+T_{\mathrm{e}}^{-}\right)\right] \\
& +\sigma_{0}\left[\bar{n}(z)-\frac{1}{2}\left(n^{+}+n^{-}\right)\right] .
\end{aligned}
$$

We now use the boundary conditions at the magnetic presheath entrance in order to determine the value of $\phi^{ \pm}$. We 
shall assume that the time-average current towards the wall at the magnetic presheath entrance, $j_{m}$, is approximately zero, so that quasi-neutrality is ensured in the main plasma. This current is $j_{m}=j_{||} \sin \alpha+j_{\perp w} \cos \alpha$, where $j_{\|}=e n\left(v_{\| \mathrm{i}}-v_{\| \mathrm{e}}\right)$ is the parallel current at the magnetic presheath entrance, $\alpha$ is the angle between the magnetic field line and the wall, and $j_{\perp w}=e n\left(v_{\perp w, \mathrm{i}}-v_{\perp w, \mathrm{e}}\right)$ is the component of the perpendicular current at the magnetic presheath entrance that is directed towards the wall. Imposing the condition $j_{m}=0$ at the magnetic presheath entrance by using the boundary conditions derived in [9], which are listed in section 3, we find that the electrostatic potential at both ends of the field line must satisfy

$$
e \phi^{ \pm} \simeq\left(\Lambda \pm \frac{\theta_{T_{\mathrm{e}}}}{2}-\theta_{n}\right) T_{\mathrm{e}}^{ \pm},
$$

where for a generic quantity $A$ we define $\theta_{A}=\rho_{\mathrm{s}} /\left(2 L_{\mathrm{A}} \tan \alpha\right)$, $\rho_{\mathrm{s}}$ is the ion sound larmor radius, and $L_{\mathrm{A}}$ is the radial equilibrium scale length of $\mathrm{A}$. Taking typical SOL parameters, e.g. $\rho_{\mathrm{s}} / L_{T} \simeq 10^{-2}$ and $\alpha=0.03 \simeq 2^{\circ}$ [11], we have that $\theta_{T_{\mathrm{e}}} \simeq 0.1$, and similarly for $\theta_{n}$. We therefore expect that the electrostatic potential at both ends of a magnetic field line will be approximately $e \phi^{ \pm} \simeq \Lambda T_{\mathrm{e}}^{ \pm}$. We remark that the electrostatic potential is measured with respect to the wall potential, which is assumed to be zero. We can thus write equation (9) as

$$
\begin{aligned}
e \bar{\phi}(z)= & \frac{1}{2} \Lambda\left(T_{\mathrm{e}}^{+}+T_{\mathrm{e}}^{-}\right)+1.71\left[\bar{T}_{\mathrm{e}}(z)-\frac{1}{2}\left(T_{\mathrm{e}}^{+}+T_{\mathrm{e}}^{-}\right)\right] \\
& +\sigma_{0}\left[\bar{n}(z)-\frac{1}{2}\left(n^{+}+n^{-}\right)\right] .
\end{aligned}
$$

Equation (11) is an analytical relation between the equilibrium electrostatic potential, the equilibrium electron temperature and density in the SOL, $\bar{\phi}=\bar{\phi}\left(\bar{T}_{\mathrm{e}}, \bar{n}\right)$. The first term on the right hand side of equation (11) represents the effect of the sheath in determining the value of $\bar{\phi}$, while the second and third terms correspond to the effect of the bulk dynamics.

We now discuss a few interesting limits of equation (11). First, in the limit of constant density and temperature along the field line, $\bar{T}_{\mathrm{e}}(z) \equiv T_{0}$ and $\bar{n}(z) \equiv n_{0}$, equation (11) implies that $\bar{\phi}$ is constant as well, more precisely:

$$
e \bar{\phi}(z)=\Lambda T_{0},
$$

and therefore, in this particular limit, the electrostatic potential is exclusively determined by the sheaths. Equation (12) is the widely used relation justifying the estimate of the radial electric field as $E_{r}=-3 \partial_{r} T_{\mathrm{e}} / e$. However, this is a slightly unrealistic limit, since even in the sheath-limited regime, where the temperature is about constant along the field lines, the density always drops when approaching the wall due to the sink action of the sheaths [4].

Second, another interesting limit of equation (11) is the case $T_{\mathrm{e}}^{+}=T_{\mathrm{e}}^{-}=0$ and $n^{+}=n^{-}=0$, namely the case where both the temperature and density drop substantially when approaching the walls. This corresponds to the detached regime, where the plasma strongly recombines and cools down before interacting with the walls [4]. In this particular limit, equation (11) gives

$$
e \bar{\phi}(z)=1.71 \bar{T}_{\mathrm{e}}(z)+\sigma_{0} \bar{n}(z),
$$

and therefore the electrostatic potential is exclusively determined by the value of density and temperature in the plasma bulk.

Third, we may assume arbitrary density and temperature profiles with a constant ratio $\sigma(z) \simeq \sigma_{0}$. This is a reasonable assumption at least if the particle and heat sources in the SOL have similar locations, a situation that is encountered in low-recycling regimes where most of the plasma in the SOL is refuelled by the core. Under this assumption, equation (11) gives

$e \bar{\phi}(z)=\frac{1}{2} \Lambda\left(T_{\mathrm{e}}^{+}+T_{\mathrm{e}}^{-}\right)+2.71\left[\bar{T}_{\mathrm{e}}(z)-\frac{1}{2}\left(T_{\mathrm{e}}^{+}+T_{\mathrm{e}}^{-}\right)\right]$.

Equation (14) is a simple relation between the equilibrium electrostatic potential and the equilibrium electron temperature in the SOL. As in equation (11), the value of $\bar{\phi}$ is determined by the combined effect of the sheath and the bulk dynamics. Their relative importance depends on the magnitude of the temperature drop established between the bulk and the sheaths. More precisely, we can write equation (14) as

$$
e \bar{\phi}(z)=\left[\Lambda f_{\mathrm{sh}}+2.71\left(1-f_{\mathrm{sh}}\right)\right] \bar{T}_{\mathrm{e}}(z),
$$

where we have defined

$$
f_{\mathrm{sh}}(z)=\frac{T_{\mathrm{e}}^{+}+T_{\mathrm{e}}^{-}}{2 \bar{T}_{\mathrm{e}}(z)} .
$$

If the temperature is constant along the field line then $f_{\text {sh }}=1$, whereas $f_{\text {sh }} \rightarrow 0$ if the temperature drops substantially when approaching the walls. These two limits are roughly representative of the sheath-limited and detached regimes, respectively. In fact, equation (12) is retrieved in the limit $f_{\text {sh }}=1$, while equation (13) is retrieved for $f_{\text {sh }} \rightarrow 0$ in the case where $\sigma(z) \simeq \sigma_{0}$.

We would like to remark that since $\Lambda$ is very close to 2.71 for hydrogen, equation (15) approximately gives $\bar{\phi}(z)=3 \bar{T}_{\mathrm{e}}(z)$. This means that the widely invoked relation $E_{r} \sim-3 \partial_{r} T_{\mathrm{e}}$ should be used carefully. For example, strongly varying temperature profiles along the field line imply that the radial electric field varies accordingly, and thus it must be computed by using the local values of temperature.

\section{Simulations in SOL conditions}

The validity of the analytical prediction for the equilibrium electrostatic potential in the SOL, equation (11), is assessed in the present section by means of global, three-dimensional fluid simulations of SOL turbulence performed with the GBS code [5]. GBS is a global, three-dimensional, full- $n$, flux-driven, two-fluid code based on the drift-reduced Braginskii equations in the limit $T_{\mathrm{i}} \ll T_{\mathrm{e}}$. GBS evolves the plasma dynamics with no separation between equilibrium and fluctuating quantities, as a balance between density and heat sources, the turbulent cross-field transport produced by plasma instabilities, and the losses at the sheaths, where the magnetic field lines terminate on the walls. We remark that, at the moment, GBS simulations do not describe ionization, recombination or radiative processes. Thus we can only expect to access sheath-limited regimes where convection is the dominant 
mechanism for particle and heat transport along the field lines, and parallel gradients are relatively small. Simulations capable of describing high-recycling and detached regimes, where heat conduction becomes important and parallel gradients are larger, are planned and will represent a testbed of the analytical predictions in these regimes.

In order to progressively approach the complexity of tokamak edge simulations, the GBS code was initially developed and used to simulate the turbulent dynamics in basic plasma physics devices of increasing complexity, namely linear devices such as LAPD [12] and simple magnetized toroidal devices such as TORPEX [13, 14] and Helimak [15]. In particular, GBS simulations of the LAPD revealed the crucial role of sheath physics in determining the nature of turbulence, showing that most of the cross-field transport arises from sheath-driven Kelvin-Helmholtz instabilities [16]. GBS results were also fully validated against experimental measurements in the TORPEX device $[17,18]$. The code was further developed to simulate the SOL of circular, limited tokamak plasmas with a large aspect ratio [5]. Recently updated with a complete set of boundary conditions that are consistent with the physics at the magnetic presheath entrance [9], GBS is now capable of describing in detail the effect of the sheath on the SOL equilibrium and turbulence. Therefore GBS is an adequate tool to assess the validity of the analytical predictions for the equilibrium electrostatic potential derived in the previous section.

The system of equations evolved by GBS to study SOL turbulence is, in the electrostatic limit and with no magnetic shear,

$$
\begin{aligned}
& \frac{\mathrm{d} n}{\mathrm{~d} t}=\frac{2}{e B}\left[\hat{C}\left(p_{\mathrm{e}}\right)-e n \hat{C}(\phi)\right]-\frac{\partial\left(n V_{\| \mathrm{e}}\right)}{\partial z}+S_{n} \\
& \frac{\mathrm{d} \nabla_{\perp}^{2} \phi}{\mathrm{d} t}=\frac{2 B}{n m_{\mathrm{i}}} \hat{C}\left(p_{\mathrm{e}}\right)-V_{\| \mathrm{i}} \frac{\partial \nabla_{\perp}^{2} \phi}{\partial z}+\frac{m_{\mathrm{i}} \Omega_{\mathrm{ci}}^{2}}{e^{2} n} \frac{\partial j_{\|}}{\partial z} \\
& \frac{\mathrm{d} T_{\mathrm{e}}}{\mathrm{d} t}=\frac{4}{3} \frac{1}{e B}\left[\frac{7}{2} T_{\mathrm{e}} \hat{C}\left(T_{\mathrm{e}}\right)+\frac{T_{\mathrm{e}}^{2}}{n} \hat{C}(n)-e T_{\mathrm{e}} \hat{C}(\phi)\right] \\
& +\frac{2}{3} \frac{T_{\mathrm{e}}}{e n} 0.71 \frac{\partial j_{\|}}{\partial z}-\frac{2}{3} T_{\mathrm{e}} \frac{\partial V_{\| \mathrm{e}}}{\partial z}-V_{\| \mathrm{e}} \frac{\partial T_{\mathrm{e}}}{\partial z}+S_{\mathrm{T}} \\
& m_{\mathrm{e}} \frac{\mathrm{d} V_{\| \mathrm{e}}}{\mathrm{d} t}=-m_{\mathrm{e}} V_{\| \mathrm{e}} \frac{\partial V_{\| \mathrm{e}}}{\partial z}-\frac{T_{\mathrm{e}}}{n} \frac{\partial n}{\partial z}+e \frac{\partial \phi}{\partial z}-1.71 \frac{\partial T_{\mathrm{e}}}{\partial z}+e v j_{\|}
\end{aligned}
$$

$$
m_{\mathrm{i}} \frac{\mathrm{d} V_{\| \mathrm{i}}}{\mathrm{d} t}=-m_{\mathrm{i}} V_{\| \mathrm{i}} \frac{\partial V_{\| \mathrm{i}}}{\partial z}-\frac{1}{n} \frac{\partial p_{\mathrm{e}}}{\partial z} .
$$

Here $\mathrm{d} f / \mathrm{d} t=\partial_{\mathrm{t}} f+(1 / B)\{\phi, f\}, \nabla_{\perp}^{2}=\partial_{x}^{2}+\partial_{y}^{2}$ and $\Omega_{\mathrm{ci}}=e B / m_{\mathrm{i}}$, where we have defined the Poisson bracket as $\{f, g\}=\partial_{y} f \partial_{x} g-\partial_{y} g \partial_{x} f$. Small diffusive terms are introduced for all quantities (see [5] for their detailed expression). In particular, we remark that the parallel electron heat conductivity $\kappa_{\|}$leads to a diffusive term, $\kappa_{\|} \nabla_{\|}^{2} T_{\mathrm{e}}$, which is small in the sheath-limited regime where convection dominates over conduction. The coordinate $z$ refers to the direction along the magnetic field $B, x$ is the radial coordinate and $y$ is the coordinate perpendicular to both $x$ and $z$ (equivalent at large aspect ratio to a poloidal coordinate). In the SOL configuration, a toroidal limiter at a given poloidal location $\theta_{l}$ defines the boundaries of the system along the $y$ direction. The curvature operator is $\hat{C}=\sin \theta \partial_{x}+\cos \theta \partial_{y}$, with $\theta=$ $\theta_{l}+y / a(\theta=0$ at the low-field-side midplane, $y=0$ and $y=2 \pi a$ at the two sides of the limiter, and $a$ is the minor radius). The source terms, $S_{n}=S_{n 0} \exp \left[-\left(x-x_{\mathrm{S}}\right)^{2} / \lambda_{\mathrm{S}}^{2}\right]$ and $S_{\mathrm{T}}=S_{\mathrm{T} 0} \exp \left[-\left(x-x_{\mathrm{S}}\right)^{2} / \lambda_{\mathrm{S}}^{2}\right]$, mimic the particle and heat outflowing from the tokamak core and thus the $x>x_{\mathrm{S}}$ region is the one of interest in the simulations. Equations (17)-(21) are solved in a domain that covers the full toroidal and poloidal angles and extends radially from $x=0$ to $x=L_{x}$. Neumann boundary conditions are applied along the $x$ direction. In the poloidal direction, magnetic presheath boundary conditions are imposed at the plasma-limiter interface,

$$
\begin{aligned}
V_{\| \mathrm{i}}= & c_{\mathrm{s}}\left[ \pm 1+\theta_{n} \mp \frac{1}{2} \theta_{T_{\mathrm{e}}}-\frac{2 \phi}{T_{\mathrm{e}}} \theta_{\phi}\right] \\
V_{\| \mathrm{e}}= & c_{\mathrm{s}}\left[ \pm \exp \left(\Lambda-e \phi / T_{\mathrm{e}}\right)-\frac{2 \phi}{T_{\mathrm{e}}} \theta_{\phi}+2\left(\theta_{n}+\theta_{T_{\mathrm{e}}}\right)\right] \\
\frac{\partial \phi}{\partial y}= & -\frac{m_{\mathrm{i}} c_{\mathrm{s}}}{e}\left[ \pm 1+\theta_{n} \pm \frac{1}{2} \theta_{T_{\mathrm{e}}}\right] \frac{\partial V_{\| \mathrm{i}}}{\partial y} \\
\frac{\partial n}{\partial y}= & -\frac{n}{c_{\mathrm{s}}}\left[ \pm 1+\theta_{n} \pm \frac{1}{2} \theta_{T_{\mathrm{e}}}\right] \frac{\partial V_{\| \mathrm{i}}}{\partial y} \\
\frac{\partial T_{\mathrm{e}}}{\partial y}= & 0 \\
\nabla_{\perp}^{2} \phi= & -\frac{m_{\mathrm{i}} \cos ^{2} \alpha}{e}\left[\left(1+\theta_{T_{\mathrm{e}}}\right)\left(\frac{\partial V_{\| \mathrm{i}}}{\partial y}\right)^{2}\right. \\
& \left.+c_{\mathrm{s}}\left( \pm 1+\theta_{n} \pm \theta_{T_{\mathrm{e}}} / 2\right) \frac{\partial^{2} V_{\| \mathrm{i}}}{\partial y^{2}}\right]
\end{aligned}
$$

A detailed derivation of this set of boundary conditions can be found in [9]. We note that while the boundary condition for the electrostatic potential, equation (24), does not impose $\phi=\Lambda T_{\mathrm{e}}$, the latter is expected to fluctuate around this value to ensure that the average current to the wall is essentially zero.

Finally, GBS works with normalized quantities by defining an arbitrary reference temperature $T_{\mathrm{e} 0}$, density $n_{0}$ and magnetic field $B_{0}$. In particular, the electron temperature and the electrostatic potential are normalized such that $T_{\mathrm{e}} \rightarrow T_{\mathrm{e}} / T_{\mathrm{e} 0}$ and $\phi \rightarrow e \phi / T_{\mathrm{e} 0}$, and analogously for the density, $n \rightarrow n / n_{0}$. The perpendicular coordinates $x$ and $y$ are normalized with respect to $\rho_{\mathrm{s} 0}=c_{\mathrm{s} 0} / \Omega_{\mathrm{ci} 0}$, where $c_{\mathrm{s} 0}=\sqrt{T_{\mathrm{e} 0} / m_{\mathrm{i}}}$ and $\Omega_{\mathrm{ci} 0}=e B_{0} / m_{\mathrm{i}}$.

For the simulations presented below, a toroidal limiter is located on the high field side midplane, i.e. $\theta_{l}=\pi$, and we use the following model parameters: major radius $R=500 \rho_{\mathrm{s} 0}$, aspect ratio $a / R \approx 0.25$, radial extension $L_{x}=100 \rho_{\mathrm{s} 0}$, and safety factor $q=4$. Thus the angle $\alpha$ between the magnetic field and the limiter is such that $\tan \alpha=a / q R \approx 0.0625$, corresponding to $\alpha \approx 3.6^{\circ}$. The ion-to-electron mass ratio is $m_{\mathrm{i}} / m_{\mathrm{e}}=200$. The particle and heat sources are radially localized at $x_{\mathrm{s}}=35 \rho_{\mathrm{s} 0}$. The resistivity $v$ is such that the electron mean free path satisfies $\lambda_{\mathrm{e}} / L_{\|} \simeq 1 / 70 \ll 1$.

Figure 1 shows typical snaphots of plasma turbulence in a poloidal cross-section for the fields of interest here. For this simulation, we have used $\Lambda=3$ for the sheath boundary conditions, approximately corresponding to the value for 


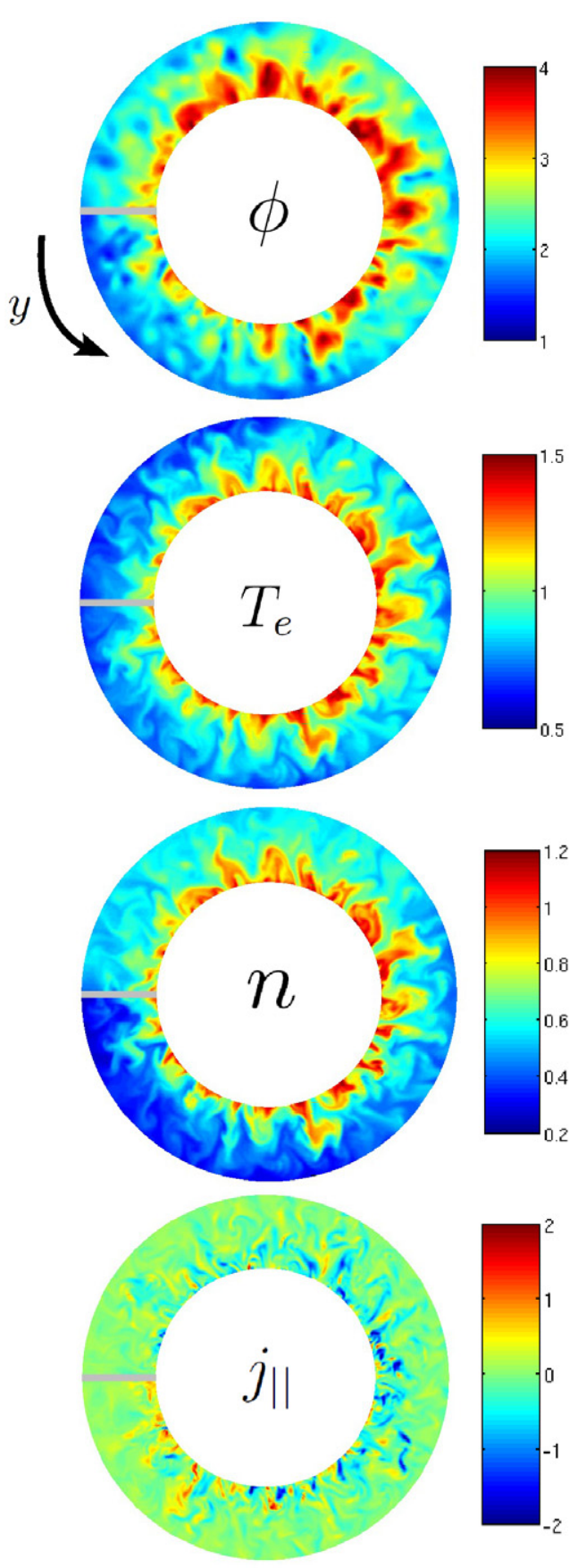

Figure 1. Snapshots in a poloidal cross-section of the electrostatic potential $\phi$, the electron temperature $T_{\mathrm{e}}$, the density $n$, and the parallel current $j_{\|}$. Results are obtained from the GBS simulations of a limited tokamak SOL, as described in section 3. The limiter plate is located at $\theta_{l}=\pi$. The snaphot covers the radial extension $\left(x_{\mathrm{s}}, L_{x}\right)$.

hydrogen. In this simulation, the time-averaged Ohm's law is fairly well described by the balance given by equation (2). In fact, figure 2 shows that the first term in equation (2), namely the parallel electric field force, is almost perfectly balanced by the sum of the pressure and thermal forces. Also, the difference between the two can be explained by the finite effect of the resistivity. This is due to the fact that the condition $\sqrt{m_{\mathrm{e}} / m_{\mathrm{i}}} \ll \lambda_{\mathrm{e}} / L_{\|}$is not exactly satisfied. We can nevertheless conclude that equation (2) is a very reasonable approximation.

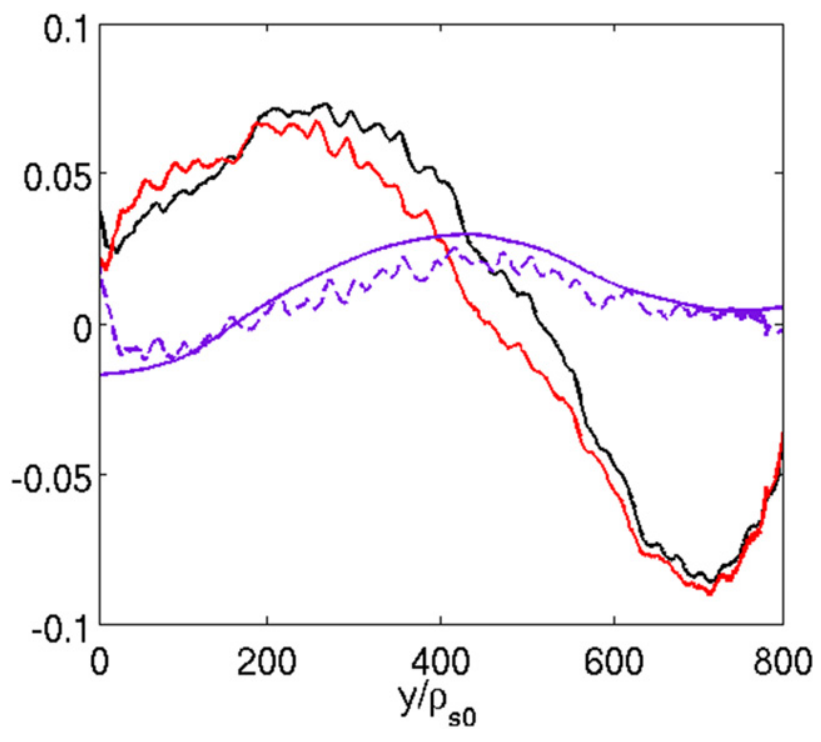

Figure 2. Balance of the dominant terms in the time-averaged Ohm's law at a given radial location $x=50 \rho_{\mathrm{s} 0}$, as a function of the poloidal coordinate. The parallel electric field force term $e \nabla_{\|} \bar{\phi}$ (black) is almost balanced by the sum of the terms related to pressure and thermal forces, $\bar{T}_{\mathrm{e}} \nabla_{\|} \bar{n} / \bar{n}+1.71 \nabla_{\|} \bar{T}_{\mathrm{e}}$ (red). The difference of the two is shown in dashed-violet. The resistivity term $\nu \bar{j}_{\|} / \bar{n}$ (solid-violet) accounts for this difference.

We now assess the validity of the analytical prediction for the equilibrium electrostatic potential, equation (11). Since the system is toroidally symmetric, the equilibrium quantities only depend on $x$ and $y$ and thus the results are shown in a poloidal cross-section. Figure 3 (left column) shows the time-averaged electrostatic potential as given by the GBS simulation, and compares it with the prediction of equation (11). Also, the widely used expression $\bar{\phi}=\Lambda T_{0}$, equation (12), is shown for comparison. The prediction of equation (11) agrees rather well with the simulation result, as it is able to capture both the magnitude and the radial and poloidal structure of the electrostatic potential. The relation $\bar{\phi}=\Lambda T_{0}$ does not capture so well the poloidal structure of the potential, but it nevertheless gives the correct order of magnitude. We note that this could be due to the fact that $\Lambda$ is comparable to 2.71 , thus the agreement being a simple coincidence. However, as shown in figure 3 (middle and right columns), simulations with an artificially high value of $\Lambda$ show that $\bar{\phi}=\Lambda T_{0}$ is also a reasonable prediction for the order of magnitude of $\bar{\phi}$. In fact, in the sheath-limited simulations presented herein, the parallel gradients are not very large and $f_{\text {sh }} \approx 0.8$, thus the effect of the sheath is expected to play a dominant role in setting the value of $\bar{\phi}$ in the SOL.

\section{Conclusions}

The electrostatic potential in an open-field-line plasma configuration, e.g. in the SOL of tokamaks, is set by the combined effect of two different mechanisms. On the one hand, the sheath physics regulates the value of $\phi$ at the end of the field lines to ensure quasi-neutrality in the main plasma. On the other hand, the electron adiabaticity sets the parallel electric field in the main plasma. We have 

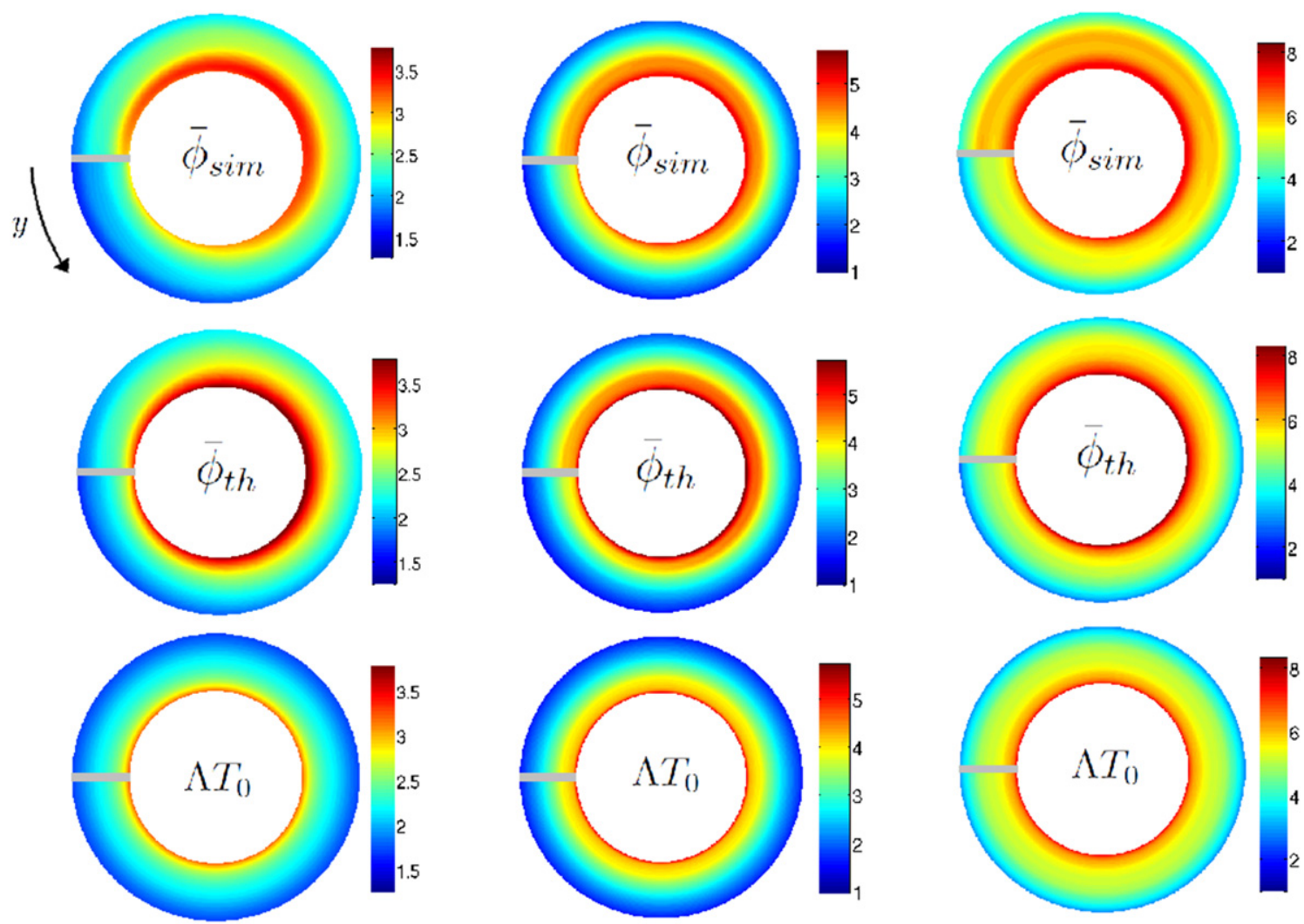

Figure 3. Equilibrium profile of the electrostatic potential $\bar{\phi}$ in a poloidal cross-section as given from GBS simulations (top row), from equation (11) (middle row), and from the widely used estimate $\bar{\phi}=\Lambda T_{0}$ (bottom row) with $T_{0}=\left(T_{\mathrm{e}}^{+}+T_{\mathrm{e}}^{-}\right) / 2$. Here $\Lambda=3$ (left column), $\Lambda=6$ (middle column), and $\Lambda=10$ (right column).

provided a general analytical relation between the equilibrium electrostatic potential and the equilibrium electron temperature and density, $\bar{\phi}=\bar{\phi}\left(\bar{T}_{\mathrm{e}}, \bar{n}\right)$, which implies that the relative importance of the two mechanisms in setting the value of $\bar{\phi}$ depends on the density and temperature drops that are established between the bulk plasma and the sheaths. This suggests that one must be careful when estimating the radial and poloidal electric fields in the SOL of tokamaks, as it may depend on the particular regime of operation: sheath-limited regime, low and high-recycling regimes, or detached regime. We would like to remark that the equilibrium radial electric field plays a crucial role in setting the plasma poloidal rotation and turbulence suppression, if the shear flow becomes sufficiently large. Moreover, the poloidal flows are recirculated at the sheath in the parallel direction [9], which in turn can lead to the generation of plasma toroidal flows [19].

The validity of equation (11) has been assessed via global, three-dimensional SOL turbulence simulations in the sheath-limited regime. Future simulations of high-recycling and detached regimes will be a good testbed of our analytical predictions.

\section{Acknowledgments}

We acknowledge useful discussions with Fabio Avino, Alexandre Bovet, Ambrogio Fasoli, Ivo Furno and Barrett Rogers. The simulations presented herein were carried out using the HELIOS supercomputer system at the Computational Simulation Centre of the International Fusion Energy
Research Centre (IFERC-CSC), Aomori, Japan, under the Broader Approach collaboration between Euratom and Japan, implemented by Fusion for Energy and JAEA. This research was supported by the Swiss National Science Foundation.

(C) Euratom 2013.

\section{References}

[1] Loarte A et al 2007 Nucl. Fusion 47 S203

[2] Shimada M et al 2007 Nucl. Fusion 47 S1

[3] LaBombard B et al 2008 Phys. Plasmas 15056106

[4] Stangeby P 2000 The Plasma Boundary of Magnetic Fusion Devices (Bristol: Institute of Physics Publishing)

[5] Ricci P et al 2012 Plasma Phys. Control. Fusion 54124047

[6] Mosetto A, Halpern F, Jolliet S and Ricci P 2012 Phys. Plasmas 19112103

[7] Ricci P and Rogers B N 2013 Phys. Plasmas 20010702

[8] Rogers B N, Drake J F and Zeiler A 1998 Phys. Rev. Lett. 814396

[9] Loizu J, Ricci P, Halpern F and Jolliet S 2012 Phys. Plasmas 19122307

[10] Zeiler A, Drake J F and Rogers B N 1997 Phys. Plasmas 42134

[11] LaBombard B et al 2004 Nucl. Fusion 441047

[12] Gekelman W et al 1991 Rev. Sci. Instrum. 622875

[13] Fasoli A et al 2006 Phys. Plasmas 13055902

[14] Fasoli A et al 2010 Plasma Phys. Control. Fusion 52124020

[15] Gentle K W and Huang H 2008 Plasma Sci. Technol. 10284

[16] Rogers B N and Ricci P 2010 Phys. Rev. Lett. 104225002

[17] Ricci P et al 2009 Phys. Plasmas 16055703

[18] Ricci P et al 2011 Phys. Plasmas 18032109

[19] Loizu J et al 2013 Nucl. Fusion submitted 